\title{
COORDINATES TRANSFORMATIONS OF THE PANORAMIC PHOTOGRAPHS ONTO A SPHERE
}

\section{Abdel-A'L M. Abdel-Wahed, Youssef A. Abbas, Farrag A. Farrag and Mohammed S. Sedek}

Civil Engineering Department, Assiut University, Assiut, Egypt

(Received January 17, 2009 Accepted February16, 2009)

\begin{abstract}
Photography of the Earth onto tangent and tilted planes and its transformation to panoramic photographs from an external exposure station, if done mathematically, is useful for interpreting the photos taken by a camera mounted on a space vehicle located anywhere in space and pointed towards any part of the Earth. General formulas for vertical and oblique photographs have been established which will be used to find the panoramic coordinates for different points on the Earth. Inverse formulas of transformation are included. A numerical example is given.
\end{abstract}

KEYWORDS: Panoramic coordinates, panoramic photos, transformation.

\section{INTRODUCTION}

A panoramic photograph is a picture of a strip of terrain taken transverse to the direction of flight. The exposure is made by a specially designed camera which scans laterally from one side of the flight path to the other. The lateral scan angle may be as great as $180^{\circ}$, in which case the photograph contains a panorama of the terrain from horizon to horizon.

By observation of parts of the Earth from certain outer point, which is the camera lens and whose altitude $\mathrm{h}$ above the Earth, we can determine their geographic coordinates $\phi$ and $\lambda$, from knowing their coordinates on panoramic photos. Special case of polar panoramic photos is given also. A single numerical example is given for all the forward and inverse coordinate transformations.

\section{The Coordinates onto the Tangent Plane}

Figure (1) shows the geometry of the oblique panoramic photograph of the reference sphere with radius $R$, the camera lens $L$ is at altitude $h$ above the earth. The principal axis $L O$ is perpendicular to the sphere and intersecting its surface at point $P_{o}$ "principal point" at geodetic latitude $\phi_{o}$ and geodetic longitude $\lambda_{o}$. Through $P_{o}$ a tangent plane $T$ to the sphere is drawn. A central projection of a point $P(\phi, \lambda)$ on the sphere onto $T$ is formed by mapping the point with the intersection $P_{l}$ of the line $L P$ with $T$.

At first the relative coordinates of $P$ to $P_{o}$ are to be calculated. They are:

The zenithal distance measured as the $\operatorname{arc} P_{o} P$ and represented by the central angle $\delta=\operatorname{angle}\left(P_{o} O P\right)$, and the geographic azimuth $\alpha$; that is the spherical angle $N P_{o} P$ at $P_{o}$, measured from the meridian $\lambda_{o}$. 


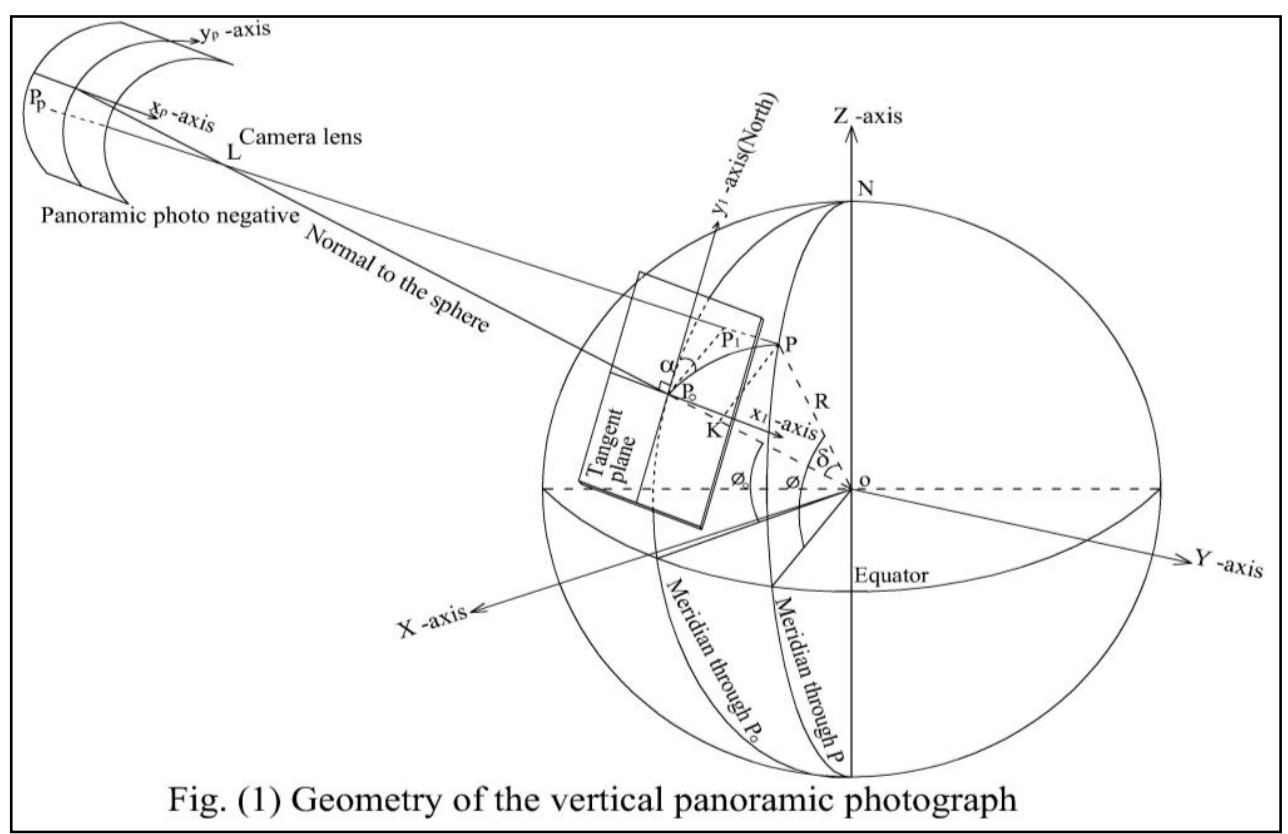

In the tangent plane $T$, let the assumed system of rectangular coordinates $\left(x_{1}\right.$, $\left.y_{1}\right)$ be such that the $y_{l}$-axis coincides with the central meridian through $P_{o}$ and directed toward the North, while the $x_{l}$-axis is perpendicular to the $y_{l}$-axis, positive east.

Figure (2) shows the polar coordinates $(r, \alpha)$ of the point $P_{l}$ onto the plane $T$.

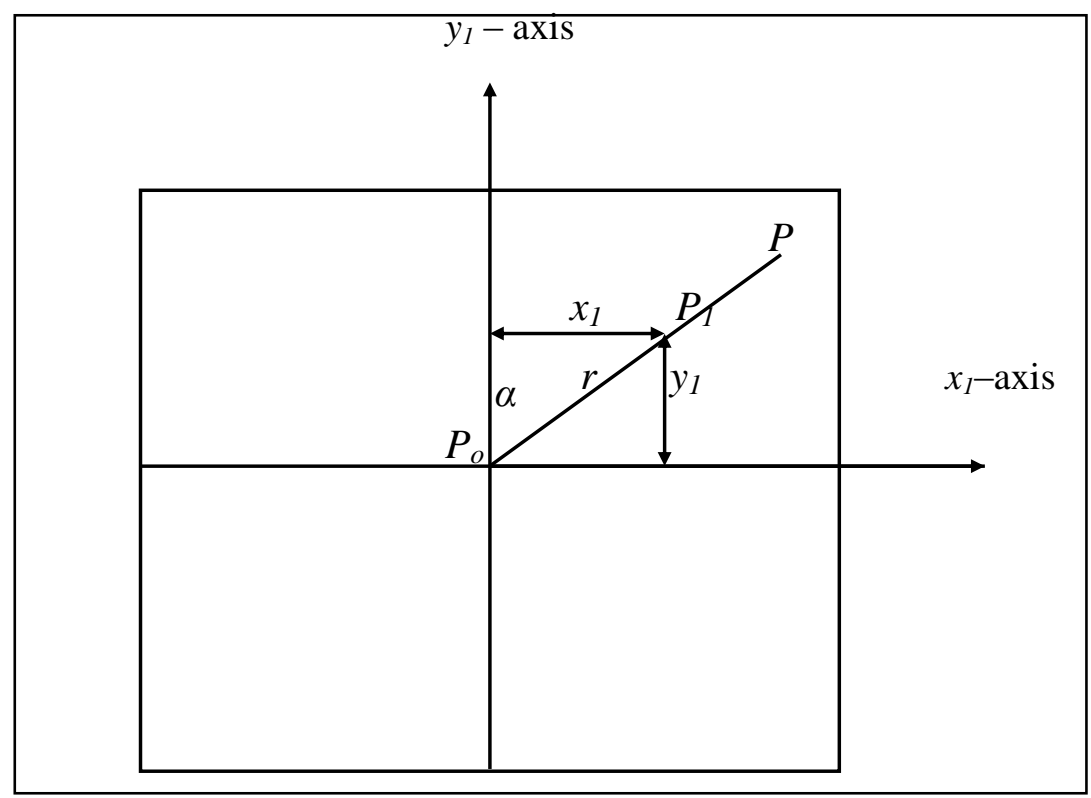

Fig. (2) Systems of rectangular and polar coordinates of the plane $T$.

Since $\alpha$ is the geographic azimuth, and $r$ is the distance $P_{o} P_{l}$ (the projection of $\operatorname{arc} P_{o} P$ on the plane), the coordinates $\left(x_{1}, y_{1}\right)$ are determined as follows: 
In the plane of arc $P_{o} P$, defined by the rays $L P_{o} O$ and $L P_{1} P$, and due to the similarity of the triangles $L P K$ and $L P_{l} P_{o}$, we get:

$$
\frac{r}{R \sin \delta}=\frac{h}{h+R(1-\cos \delta)}
$$

Since,

$$
\begin{aligned}
& x_{1}=r \sin \alpha \\
& y_{1}=r \cos \alpha,
\end{aligned}
$$

and,

then substituting from equation (1), we obtain:

$$
\begin{aligned}
& x_{1}=\frac{h \sin \delta \sin \alpha}{\left(1+\frac{h}{R}\right)-\cos \delta} \\
& y_{1}=\frac{h \sin \delta \cos \alpha}{\left(1+\frac{h}{R}\right)-\cos \delta}
\end{aligned}
$$

Applying the sine law to the spherical triangle $N P P_{o}$, shown in Figure (3), we get:

$$
\frac{\sin d \lambda}{\sin \delta}=\frac{\sin \alpha}{\sin \left(\frac{\pi}{2}-\phi\right)}=\frac{\sin \alpha}{\cos \phi},
$$

hence, $\quad \sin \delta \sin \alpha=\cos \phi \sin d \lambda$.

$\sin \delta \cos \alpha=\sin \phi \cos \phi_{o}-\cos \phi \sin \phi_{o} \cos d \lambda$

The cosine formula for the side $\delta$ gives:

$$
\cos \delta=\sin \phi \sin \phi_{o}+\cos \phi \cos \phi_{o} \cos d \lambda
$$

Substituting a, b \& c into (2) and (3), we obtain; after some algebraic reductions:

$$
\begin{aligned}
& x_{1}=h \frac{C \sin d \lambda}{G-S \sin \phi_{o}-C \cos \phi_{o} \cos d \lambda} \\
& y_{1}=h \frac{S \cos \phi_{o}-C \sin \phi_{o} \cos d \lambda}{G-S \sin \phi_{o}-C \cos \phi_{o} \cos d \lambda}
\end{aligned}
$$

Where,

$$
\begin{aligned}
& C=\cos \phi \\
& S=\sin \phi \\
& G=\left(1+\frac{h}{R}\right)
\end{aligned}
$$

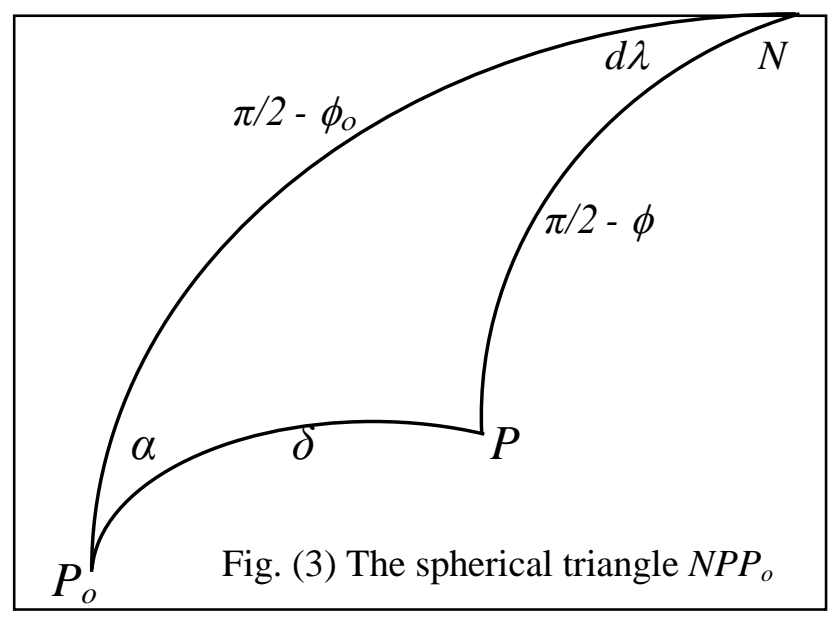


These equations represent the plane rectangular coordinates of the projection of any point on the sphere onto the tangent plane in terms of the geodetic coordinates $(\phi, \lambda)$. Replacing $d \lambda$ by its value $\left(\lambda-\lambda_{o}\right)$, that is $\lambda$ for $P$ and $\lambda_{o}$ for $P_{o}$, we obtain the general formulas of the coordinates for the image of a point on the sphere onto a tangent plane:

$$
\begin{aligned}
& x_{1}=h \frac{C \cos \lambda_{o} \sin \lambda-C \sin \lambda_{o} \cos \lambda}{G-S \sin \phi_{o}-C \cos \phi_{o} \cos \lambda_{o} \cos \lambda-C \cos \phi_{o} \sin \lambda_{o} \sin \lambda} \\
& y_{1}=h \frac{S \cos \phi_{o}-C \sin \phi_{o} \cos \lambda_{o} \cos \lambda-C \sin \phi_{o} \sin \lambda_{o} \sin \lambda}{G-S \sin \phi_{o}-C \cos \phi_{o} \cos \lambda_{o} \cos \lambda-C \cos \phi_{o} \sin \lambda_{o} \sin \lambda}
\end{aligned}
$$

\section{Rotation of the Coordinate Axes}

We considered in the tangent plane that the $y_{l}$-axis is due North. This is not always the case, so we'll rotate the coordinate axes $\left(x_{1}, y_{1}\right)$ in the tangent plane to the axes $\left(x_{2}, y_{2}\right)$. The $y_{2}$-axis makes clockwise angle $\gamma$ with the North direction. The new coordinates $x_{2}$ and $y_{2}$ of $P_{1}$ are:

$$
\begin{aligned}
& x_{2}=x_{1} \cos \gamma-y_{1} \sin \gamma \\
& y_{2}=x_{1} \sin \gamma+y_{1} \cos \gamma
\end{aligned}
$$

\section{Projection onto the Tilted Plane}

Let $P$ be projected through $L$ onto a tilted plane $I$ through $x_{2}$-axis. As shown in Figure (4), the tilted plane $I$ makes an angle $t$ with plane $T$. The coordinates $\left(x_{3}, y_{3}\right)$ of the projection $P_{2}$ in the tilted plane $I$, with the origin of axes at $P_{o}\left(\phi_{o}, \lambda_{o}\right)$ are to be found. Let the $x_{3}$-axis lie at the intersection of the two planes $T$ and $I$ (i.e. $x_{3}=x_{2}$ ), then the $y_{3^{-}}$ axis lies in the principal plane $L P_{o} y_{2}$. The rectangular coordinates $\left(x_{3}, y_{3}\right)$ of $P_{2}$ on the plane $I$ depend on the coordinates $\left(x_{2}, y_{2}\right)$. From the triangle $P_{1} P_{2} P_{o}$, we get [8]:

$$
\begin{aligned}
& y_{3}=h y_{2} /\left(h \cos t+y_{2} \sin t\right) \\
& x_{3}=h \cos t x_{2} /\left(h \cos t+y_{2} \sin t\right)
\end{aligned}
$$

\section{Translation and Rotation of the Axes onto the Tilted Plane}

On the inclined space-photographs, $P_{o}$ can't be precisely located, while center $V_{I}$ along the optical axis, can be located if the picture has been adjusted for this aim. So the need arises to translate the origin of the axes from $P_{o}$ to $V_{I}$ along the ordinate axis $P_{o} y_{3}$, as shown in Figure (4).

Consequently, all ordinates from equation (13) become shorter by distance $\left(P_{o} V_{I}=h \sin t\right)$, while the abscissas remain unchanged. The point $P_{2}$ has now the translated coordinates $x_{4} \& y_{4}$ as follows:

$$
\begin{aligned}
& x_{4}=x_{3} \\
& y_{4}=y_{3}-h \sin t
\end{aligned}
$$

If the $x_{4}, y_{4}$ coordinates are rotated by an angle $\theta$ around $V_{I}$ in the plane $I$, and if the $x$ and $y$ are the new axes, as shown in Figure (4), then the rectangular coordinates of $P_{2}$ related to this system, become:

$$
\begin{aligned}
& x=x_{4} \cos \theta-y_{4} \sin \theta \\
& y=x_{4} \sin \theta+y_{4} \cos \theta
\end{aligned}
$$




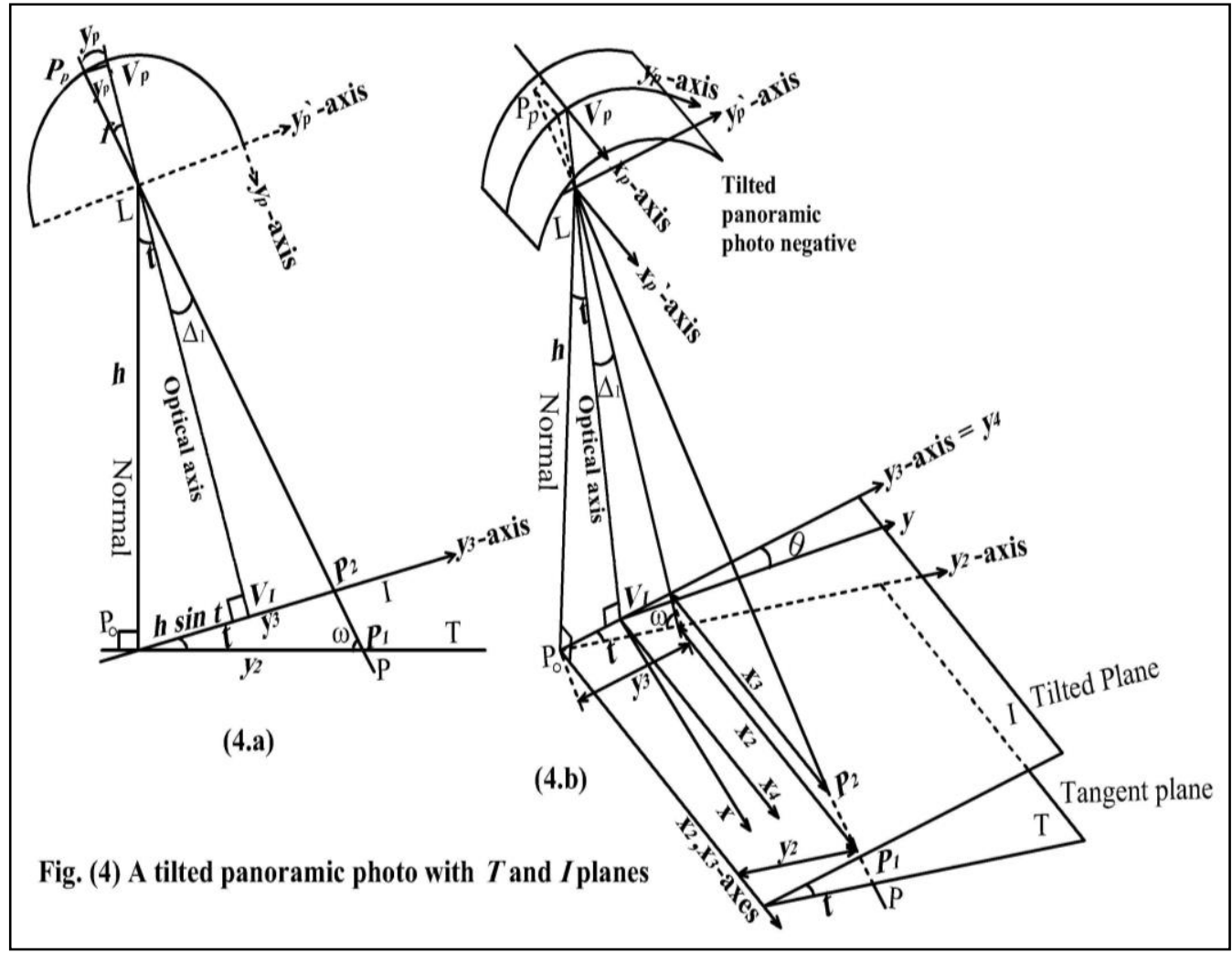

After we group the constants terms from those containing $\phi$ and $\lambda$, we reach to the synthetical formulas of all the previous changes of the whole system of photos of points on the sphere onto a plane indicated when we know the parameters:

$$
\begin{aligned}
& x=\frac{c_{1} X+c_{2} Y+c_{3} Z+c_{4}}{c_{5} X+c_{6} Y+c_{7} Z+1} \\
& y=\frac{c_{8} X+c_{9} Y+c_{10} Z+c_{11}}{c_{5} X+c_{6} Y+c_{7} Z+1}
\end{aligned}
$$

Where, $\quad X=C \cos \lambda$

$$
\begin{aligned}
& Y=C \sin \lambda \quad \text {, where } C, S \text { are } \cos \phi \text { and } \sin \phi \text { respectively. } \\
& Z=S \quad l
\end{aligned}
$$

The expressions of the eleven parameters required to calculate the rectangular coordinates on the space-photographs or its mathematical model, $x$ and $y$, when the geographical coordinates $\phi \& \lambda$ are given, are as follows:

$$
\begin{aligned}
& c_{1}=\left[\left(K_{2} \cos \theta-K_{6} \sin \theta\right) h \cos t\right] / K_{12} \\
& c_{2}=\left[\left(K_{1} \cos \theta-K_{5} \sin \theta\right) h \cos t\right] / K_{12} \\
& c_{3}=\left[\left(K_{3} \cos \theta-K_{7} \sin \theta\right) h \cos t\right] / K_{12} \\
& c_{4}=-K_{8} h \sin \theta \cos t / K_{12} \\
& c_{5}=K_{10} / K_{12} \\
& c_{6}=K_{9} / K_{12} \\
& c_{7}=K_{11} / K_{12} \\
& c_{8}=\left[\left(K_{2} \sin \theta+K_{6} \cos \theta\right) h \cos t\right] / K_{12}
\end{aligned}
$$




$$
\begin{aligned}
c_{9} & =\left[\left(K_{1} \sin \theta+K_{5} \cos \theta\right) h \cos t\right] / K_{12} \\
c_{10} & =\left[\left(K_{3} \sin \theta+K_{7} \cos \theta\right) h \cos t\right] / K_{12} \\
c_{11} & =K_{8} h \cos \theta \cos t / K_{12}
\end{aligned}
$$

where, $K_{1}=\cos \lambda_{o} \cos \gamma+\sin \phi_{o} \sin \lambda_{o} \sin \gamma$

$K_{2}=-\sin \lambda_{o} \cos \gamma+\sin \phi_{o} \cos \lambda_{o} \sin \gamma$

$K_{3}=-\cos \phi_{o} \sin \gamma$

$K_{5}=\cos \lambda_{o} \sin \gamma-\sin \phi_{o} \sin \lambda_{o} \cos \gamma$

$K_{6}=-\sin \lambda_{o} \sin \gamma-\sin \phi_{o} \cos \lambda_{o} \cos \gamma$

$K_{7}=\cos \phi_{o} \cos \gamma$

$K_{8}=-G \sin t$

$K_{9}=K_{5} \sin t-\cos \phi_{o} \sin \lambda_{o} \cos t$

$K_{10}=K_{6} \sin t-\cos \phi_{o} \cos \lambda_{o} \cos t$

$K_{11}=K_{7} \sin t-\sin \phi_{o} \cos t$

$K_{12}=G \cos t$

$K_{5}{ }^{`}=K_{5} \cos t+\cos \phi_{o} \sin \lambda_{o} \sin t$

$K_{6}{ }^{`}=K_{6} \cos t+\cos \phi_{o} \cos \lambda_{o} \sin t$

$K_{7}{ }^{\prime}=K_{7} \cos t+\sin \phi_{o} \sin t$

In equations (19) and (20), the constants in the denominators are dimensionless, but those in the numerators have the same units as $h$ and $R$. By these formulas as well as with their numberless variants, we can transform the geographical coordinates of points onto the Earth or any planet in rectangular coordinates of the corresponding point on the space-photographs or pseudo-space-photographs.

\section{FINAL COORDINATES TRANSFORMATION TO THE TILTED PANORAMIC PHOTOGRAPH}

In the tilted panoramic photo taken from the exposure station $L$, the $y_{p}$-axis is taken through $L$ parallel to the $y$-axis in the tilted plane $I$, and the $x_{p}$-axis is perpendicular to $y_{p}{ }^{\prime}$-axis in the direction of flight, as shown in Figure (4), the coordinates $\left(x_{p}{ }^{\prime}, y_{p}{ }^{\prime}\right)$ are determined as follows:

$$
y_{p}=f \sin \left(\tan ^{-1}\left[\frac{y}{h \cos t}\right]\right)
$$

and by similarity, we find:

$$
x_{p}{ }^{`}=\frac{x}{y} f \sin \left(\tan ^{-1}\left[\frac{y}{h \cos t}\right]\right)
$$

where, $f=$ the camera focal length.

Equations (21) and (22) permit the computation of the coordinates $\left(x_{p}{ }^{\prime}, y_{p}{ }^{\prime}\right)$ of the point $P_{p}$ in terms of the coordinates $(x, y)$ of the point $P_{2}$ onto the tilted plane $I$.

If we develop the cylindrical surface of the panoramic film, then any point $P_{p}$ on the panoramic photo will have the coordinates $\left(x_{p}, y_{p}\right)$, as shown in Figure (4), the $x_{p}$-axis is taken parallel to the $x_{p}$ '-axis in the direction of flight passing through the position of zero scan angle, and the $y_{p}$-axis is taken along the trace of the intersection of the camera lens optical axis with the film during scanning, so we have:

$$
\begin{aligned}
& x_{p}=x_{p}{ }^{\prime} \\
& y_{p}=f\left(\pi / 180^{\circ}\right) \Delta_{1}=f\left(\pi / 180^{\circ}\right) \sin ^{-1}\left(y_{p}{ }^{`} / f\right)
\end{aligned}
$$


$\Delta_{l}$ is in degree units.

These equations expressing the panoramic coordinates $\left(x_{p}, y_{p}\right)$ in terms of the coordinates $\left(x_{p}{ }^{\prime}, y_{p}{ }^{\prime}\right)$.

By substituting from equations (21), (22) into (24), (23), we get:

$$
\begin{aligned}
& x_{p}=\frac{x}{y} f \sin \left(\tan ^{-1}\left[\frac{y}{h \cos t}\right]\right) \\
& y_{p}=\frac{f \pi}{180^{\circ}} \tan ^{-1}\left[\frac{y}{h \cos t}\right]
\end{aligned}
$$

Equations (25), (26) permit the computation of the panoramic coordinates $\left(x_{p}\right.$, $\left.y_{p}\right)$ of the point $P_{p}$ in terms of the coordinates $(x, y)$ of the point $P_{2}$. It is to be noticed that, a parallel displacement of the photo in the direction of the optical axis change only the scale of the photograph.

\section{Inverse Transformations}

To calculate the coordinates $(x, y)$ of the tilted plane $I$ in terms of the coordinates $\left(x_{p}\right.$, $y_{p}$ ) of the point $P_{p}$ in the panoramic photo, from equation (26) by solving it for $y$, we obtain:

$$
y=h \cos t \tan \left[\frac{180^{\circ} y_{p}}{f \pi}\right]
$$

and from equation (25) by solving it for $x$, we get:

$$
x=\frac{y x_{p}}{f \sin \left(\tan ^{-1}\left[\frac{y}{h \cos t}\right]\right)}
$$

Substituting from equations (11) through (18), we obtain:

$$
\begin{aligned}
& x_{1}=h \frac{D_{1} y+D_{2} x+D_{3}}{h \cos ^{2} t+(x \sin \theta-y \cos \theta) \sin t} \\
& y_{1}=h \frac{D_{4} y+D_{5} x+D_{3}}{h \cos ^{2} t+(x \sin \theta-y \cos \theta) \sin t}
\end{aligned}
$$

Where,

$$
\begin{aligned}
& D_{1}=\sin \gamma \cos t \cos \theta+\cos \gamma \sin \theta \\
& D_{2}=\cos \gamma \cos \theta-\sin \gamma \cos t \sin \theta \\
& D_{3}=h \sin \gamma \cos t \sin t \\
& D_{4}=\cos \gamma \cos t \cos \theta-\sin \gamma \sin \theta \\
& D_{5}=-(\cos \gamma \cos t \sin \theta+\sin \gamma \cos \theta) \\
& D_{6}=h \cos \gamma \cos t \sin t
\end{aligned}
$$

Knowing $x_{1}$ and $y_{1}$, we can obtain the geodetic latitude $\phi$ and longitude $\lambda$, as follows:

\section{Geodetic latitude $\phi$}

From the spherical triangle $N P P_{o}$ in Figure (3), by cosine law, we obtain:

$$
\phi=\sin ^{-1}\left[\sin \phi_{o} \cos \delta+\left(y_{1} / r\right) \cos \phi_{o} \sin \delta\right]
$$

\section{Geodetic longitude $\lambda$}

From the spherical triangle $N P P_{o}$, using the sine and cosine laws, and with some algebraic reductions, we obtain: 


$$
\lambda=\lambda_{o}+\tan ^{-1}\left(\frac{x_{1}}{r \cos \phi_{o} \cot \delta-y_{1} \sin \phi_{o}}\right)
$$

Where, $\quad \sin \delta=\frac{G+\sqrt{1-\frac{r^{2}}{h R}(G+1)}}{\frac{r}{h}+\frac{h}{r}} \quad, r=\sqrt{x_{1}^{2}+y_{1}^{2}}$

\section{Special case}

\section{Polar panoramic photos (origin at $\boldsymbol{N}$ ) with $\gamma=0$ of the sphere}

The polar panoramic photographs are simple form of the general case in which the origin $P_{o}$ at the pole $N$, where $\left(\phi_{o}=\pi / 2\right)$, as shown in Figure (5).

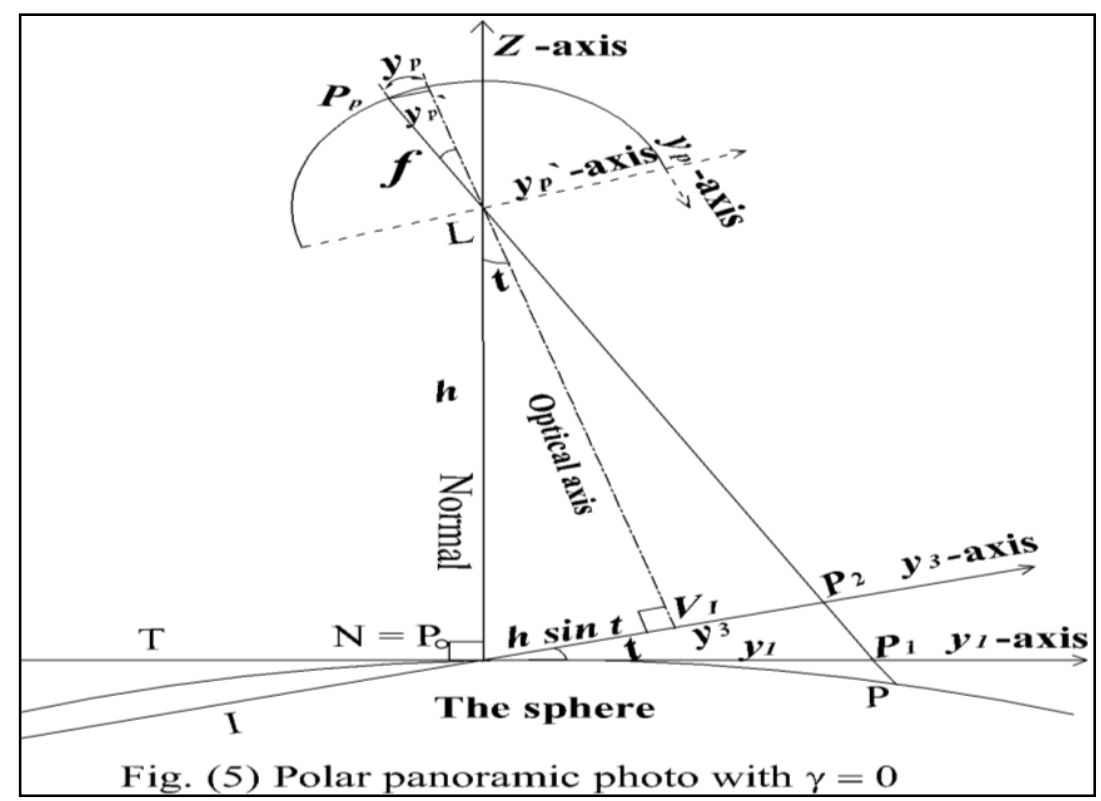

\section{The Coordinates onto the Tangent Plane T}

The coordinates $\left(x_{1}, y_{1}\right)$ in terms of $(\phi, \lambda)$ in equations (4) and (5) become:

$$
\begin{aligned}
& x_{1}=h \frac{C \sin \left(\lambda-\lambda_{o}\right)}{G-S} \\
& y_{1}=-h \frac{C \cos \left(\lambda-\lambda_{o}\right)}{G-S}
\end{aligned}
$$

\section{The Coordinates onto the Tilted Plane I}

The coordinates $\left(x_{3}, y_{3}\right)$ in terms of $(\phi, \lambda)$ become:

$$
x_{3}=h \cos t \frac{C \sin \left(\lambda-\lambda_{o}\right)}{(G-S) \cos t-C \sin t \cos \left(\lambda-\lambda_{o}\right)}
$$




$$
y_{3}=-h \frac{C \cos \left(\lambda-\lambda_{o}\right)}{(G-S) \cos t-C \sin t \cos \left(\lambda-\lambda_{o}\right)}
$$

\section{Coordinates Transformation to a Tilted Polar Panoramic Photo}

We can obtain the coordinates $\left(x_{p}{ }^{\prime}, y_{p}{ }^{\prime}\right)$ and $\left(x_{p}, y_{p}\right)$ on the panoramic photo in terms of the tilted coordinates $\left(x_{3}, y_{3}\right)$ of the point $P_{2}$, as shown in Figure (5):

$$
\begin{aligned}
& y_{p}{ }^{\prime}=f \sin \left(\tan ^{-1}\left[\frac{y_{3}-h \sin t}{h \cos t}\right]\right) \\
& x_{p}{ }^{\prime}=\frac{x_{3}}{y_{3}-h \sin t} f \sin \left(\tan ^{-1}\left[\frac{y_{3}-h \sin t}{h \cos t}\right]\right)
\end{aligned}
$$

and by substituting into (23), (24), we obtain:

$$
\begin{aligned}
& x_{p}=\frac{x_{3}}{y_{3}-h \sin t} f \sin \left(\tan ^{-1}\left[\frac{y_{3}-h \sin t}{h \cos t}\right]\right) \\
& y_{p}=\frac{f \pi}{180^{\circ}} \tan ^{-1}\left[\frac{y_{3}-h \sin t}{h \cos t}\right]
\end{aligned}
$$

\section{Numerical Example}

An example is given to calculate all the forward and inverse coordinates with the help of the equations. Given:

$R=6371000 m \quad, h=10000 \mathrm{~m} \quad, \phi_{o}=25^{\circ} \mathrm{N} . \quad, \lambda_{o}=0 \quad, \phi=30^{\circ} \mathrm{N} . \quad, \lambda=30^{\circ} \mathrm{E}$.

$\gamma=40^{\circ}$ clockwise from the North, $t=20^{\circ}, \theta=20^{\circ}$ clockwise from the positive $y_{4}$-axis.

To find $x_{l}$ and $y_{l}$ from the given $\phi$ and $\lambda$, equations (4) to (8) are used and we get:

$$
\begin{array}{lll}
C=\sqrt{0.75} & , S=0.5 & , G=1.001569612 \\
x_{1}=39176.16101 \mathrm{~m} & , y_{1}=12321.59951 \mathrm{~m}
\end{array}
$$

By rotating the axes an angle $\gamma=40^{\circ}$ clockwise from the North, the coordinates $x_{2}$ and $y_{2}$ from equations (11) and (12) are:

$$
x_{2}=22090.50895 \mathrm{~m} \quad, y_{2}=34620.84373 \mathrm{~m}
$$

To find $x_{3}$ and $y_{3}$, using equations (13) and (14), we get:

$$
x_{3}=9774.147768 \mathrm{~m} \quad, y_{3}=16301.40397 \mathrm{~m}
$$

To find $x_{4}$ and $y_{4}$ from $\left(x_{3}, y_{3}\right)$, using equations (15) and (16) we get:

$$
x_{4}=9774.147768 \mathrm{~m} \quad, y_{4}=13881.20253 \mathrm{~m}
$$

To find $x$ and $y$ from $\left(x_{4}, y_{4}\right)$ for the given $\phi \& \lambda$, using equations (17) to (20) we have:

$$
x=4779.063795 \mathrm{~m} \quad, y=15447.32639 \mathrm{~m}
$$

To calculate the panoramic coordinates $\left(x_{p}{ }^{\prime}, y_{p}{ }^{\prime}\right)$ and $\left(x_{p}, y_{p}\right)$ from $x$ and $y$, $f=0.15 m$, equations (21) to (26), yield:

$$
\begin{array}{ll}
x_{p}{ }^{`}=0.039647146 m & , y_{p}{ }^{`}=0.128151127 m \\
x_{p}=0.03964714 m & , y_{p}=0.15364218 m
\end{array}
$$


To calculate the inverse coordinates $(x, y)$ from the coordinates $\left(x_{p}, y_{p}\right)$, using equations (27) and (28), we get:

$$
y=15447.326 \mathrm{~m} \quad, x=4779.0638 \mathrm{~m}
$$

and from equations (29) and (30), we get:

$$
x_{I}=39176.161 \mathrm{~m} \quad, y_{l}=12321.6 \mathrm{~m}
$$

To find the inverse coordinates $\phi$ and $\lambda$ from $x_{I}$ and $y_{1}$, equations (31) and (32) yield:

$$
\phi=30^{\circ} \quad, \lambda=30^{\circ}
$$

with, $\quad r=41068.15562 m \quad, \sin \delta=0.45392485037 \quad, \delta=26^{\circ} 59^{`} 44.8^{\prime \prime}$

\section{CONCLUSIONS}

In this paper, photography of the celestial bodies and transformations that to panoramic photographs are discussed. This resembles the actual photographs taken by space vehicles. We considered the Earth as a sphere.

The resulting formulas for coordinates transformations $x_{l}$ and $y_{l}$ were deduced in terms of the geodetic coordinates. General formulas are established which are used to find the panoramic coordinates for different points on the Earth. The axes must be rotated clockwise angle $\gamma$ with the North direction to allow any azimuth.

The Inverse transformations permit the calculations of $\phi$ and $\lambda$ for any selected point on the photo. Special case of the polar panoramic photographs of the sphere, are derived. Finally we checked the accuracy of all the forward and inverse formulas for coordinates transformations by a numerical example.

\section{REFERENCES}

1. Wolf P. R., "Elements of photogrammetry", McGraw-Hill, Inc., 1974.

2. Maling D. H., "Coordinate Systems and Map Projections", Second Edition, Pergoman Press, Oxford, New York, 1992.

3. Maling D. H., "Measurements from Maps ", Pergoman Press, Oxford, New York, 1989.

4. Francis H. M., Edward M. M., "Photogrammetry", Third Edition, Harper and Row Inc., New York, 1980.

5. Borkowski K. M., "Transformation of Geocentric to Geodetic Coordinates without Approximations", D. Reidel Publishing Company, Astrophysics and Space Science 139, pp.1-3, 1987.

6. Schmid E., "The Earth as Viewed From Satellite", United States Government Printing Office, Washington, Technical Bulletin No.20, pp.1-21, 1962.

7. Tomas S., Larry D., "Coordinate System Used in Geodesy: Basic Definitions and Concepts", ASCE, Journal of Surveying Engineering, Vol.114, No.2, May, 1988.

8. Youssef A. , "Triple projection of a topographic surface from an external perspective center", Ph.D Thesis, Civil Engineering Department, Faculty of Engineering, Assiut University, Assiut, Egypt ,1993. 


\section{" تحويل الإحداثيات للصور البانورامية على كرة "}

الصسورة البانوراميـة هي صـورة لثريحة مـن سطح الأرض مأخوذة عرضياً لاتجـاه الطيران، حيث يتت

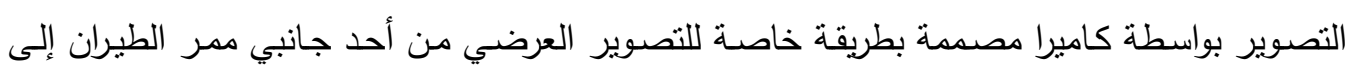

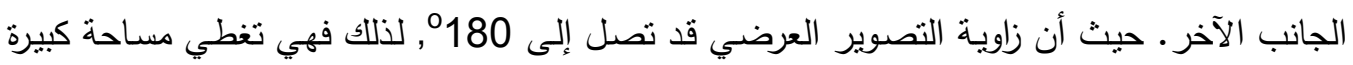
من سطح الأرض مقارنة بالصورة العادية. و في الصور البانورامية نستخدم فقط الجزء المركزي من عدسة الكاميرا, لذلك فإن درجة الوضوح للصنور مالصور البانورامية أكبر من مثيلتها للصور العادية.

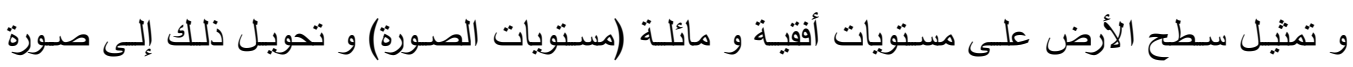

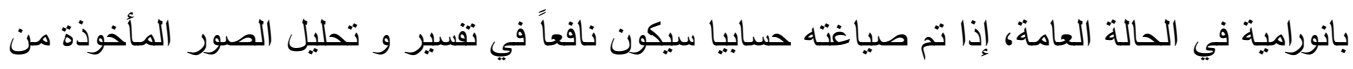

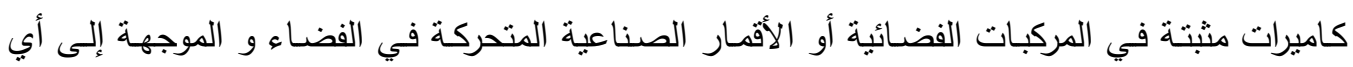

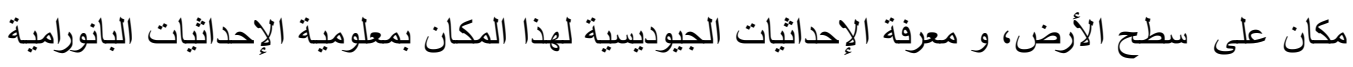
من الصورة.

و يثتنل البحث على المعادلات العكسية للتحويل بين الإحداثيات. و ويشتمل أيضاً على حالة خاصـة

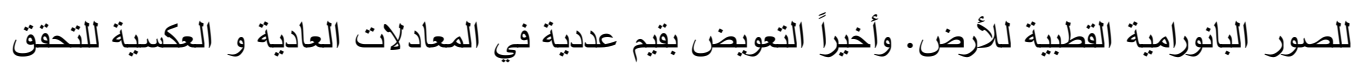
من صحتها. 